\title{
HIDDEN TREASURES UNCOVERED: SUCCESSFUL DETECTION OF FOSSILS BELOW THE SURFACE IN LARGE LIMESTONE BLOCKS USING A STANDARD MEDICAL X-RAY CT SCANNER
}

\author{
MIGUEL PRÔA ${ }^{1,2}{ }^{2}$ DANIEL POUIT' ${ }^{1}$, THOMAS ROUILLARD ${ }^{1}$, PEGGY VINCENT ${ }^{3, *},{ }^{*}$ BENOÎT MELLIER ${ }^{1}$

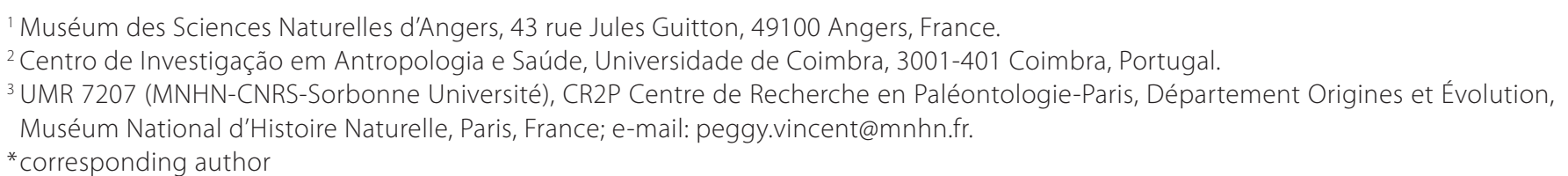

Prôa, M., Pouit, D., Rouillard, T., Vincent, P., Mellier, B. (2021): Hidden treasures uncovered: successful detection of fossils below the surface in large limestone blocks using a standard medical X-ray CT scanner. - Fossil Imprint, 77(1): 36-42, Praha. ISSN 2533-4050 (print), ISSN 2533-4069 (on-line).

\begin{abstract}
The use of CT scans in palaeontology has a long history. Most X-ray CT scans of fossils are carried out on previously prepared specimens and seldom on unprepared blocks of fossils. Here we report the use of a standard medical X-ray CT scanner to detect vertebrate and invertebrate fossils inside limestone blocks as an aid to subsequent preparation. The results were largely successful, with low-resolution images and radiodensity thresholds which nevertheless created sufficient contrast for identification of objects and their location inside blocks of limestone, thus optimizing the allocation of time and resources for palaeontological preparation. We conclude that the use of medical X-ray CT scanners for an initial visual inspection of limestone blocks for the presence of below the surface fossils is possible, cost effective and reliable. In addition, it allows the original raw data to be preserved as a digital object. The advantages of making use of standard medical X-ray CT scanners to facilitate palaeontological preparation under logistic or budgetary limitations is becoming more and more apparent.
\end{abstract}

Key words: palaeontology, fossils, X-ray CT scanning, limestone, cost effectiveness

Received: February 26, 2021 | Accepted: July 1, 2021 | Issued: December 9, 2021

\section{Introduction}

Computed tomography (CT) scanners use X-rays to visually explore the inside of an object while maintaining its physical integrity (Racicot 2017). In palaeontology, the use of X-rays to explore the inside of fossils was surprisingly precocious (Brühl 1896, Lemoine 1896), the first applications taking place just months after Röntgen's first publication (Röntgen 1895). X-ray technology in palaeontology has been used ever since, and has been recognized as being invaluable, as exemplified by the important and aesthetically superb work of Hohenstein (Hohenstein 2004). The use of X-ray CT scans in palaeontology was also attempted relatively soon (Jungers and Minns 1979, Conroy and Vannier 1984) after the technique was made available (Hounsfield 1973) and is now the most commonly applied approach for digitalisation of fossil specimens (e.g., Tafforeau et al. 2006, Cunningham et al. 2014, Sutton et al. 2014, Schilling et al. 2014, Smilg and Berger 2015, Lautenschlager 2016, Rowe et al. 2016, Racicot 2017).

Most palaeontological X-ray CT scanning is used to make 3D digital models of fossils (Sutton 2008, Herbin et al. 2010, Garcia Sanz et al. 2013), to reconstruct and restore fragile fossils digitally (Lautenschlager 2016), or to study the internal anatomy of animals, plants or even fungi (e.g., Gee 2013, Pallua et al. 2015, Maldanis et al. 2016). X-ray CT scanning is usually performed on previously prepared or partially prepared specimens (Balzeau et al. 2010, Smilg and Berger 2015) and seldom on unprepared blocks of fossils for which interest in the specimen has not been established. Fossil preparation is the process of removing fossils from the rock encasing them, but traditional manual preparation is time-consuming and often hazardous for fragile fossils: searching for fossils immediately below the surface of the rock is a blind process largely relying on the skill of the individual preparator (Smilg and Berger 2015). CT data can aid the manual preparation by guiding removal of the encasing rock matrix in a way which will avoid damage to the fossil (Conroy and Vannier 1984, Bristowe et al. 2004, Schilling et al. 2014, Smilg and Berger 2015). Additionally, since fossil preparation almost always results in destruction of the surrounding matrix, contextual information may be unavoidably lost (du Plessis et al. 2013, Smilg and Berger 
2015). One other important advantage of prior X-ray CT scanning of a block of fossils is to safeguard the unprepared block of fossils as a digital object, thus allowing for retrospective examination of data after it has been physically destroyed during preparation.

$\mathrm{X}$-ray CT scanning in palaeontology is nevertheless constrained by logistic limitations (Sutton et al. 2014). From the different types of X-ray CT scanners, those producing high-resolution images, such as laboratory X-ray $\mu \mathrm{CT}$ systems and synchrotron facilities, should ideally be favoured. Unfortunately, these powerful scanning machines are not widely available and are usually only able to scan small size specimens (from a few micrometres to approximately $50 \mathrm{~cm}$; Donoghue et al. 2006, Sutton et al. 2016). In addition, there is the financial cost linked to maintenance of the equipment. In contrast, medical X-ray CT scanners are much more widespread and with greater availability. The operative costs are comparatively low (maintenance costs of the equipment usually being covered by the medical facility to which it belongs), and the machines are designed to scan human-sized objects (Smilg 2017). Unfortunately, distribution of medical and laboratory X-ray CT systems, their availability or cost are rarely discussed in the literature (however see Cunningham et al. 2014). Medical X-ray CT systems are not, however, designed to produce high-resolution images as the radiation emitted must be kept within safe limits (Lautenschlager 2016). For this reason, medical X-ray CT scanning is less than ideal when the purpose is $3 \mathrm{D}$ modelling or detailed study of the structure of prepared fossils. But for an initial visual inspection of a fossil-bearing block of rock matrix, it may be cost effective and reliable.

Here we report the use of a standard medical X-ray CT scanner to detect fossils inside limestone blocks as an aid to subsequent preparation. A common major problem in this approach is the lack of sufficient radiodensity contrast between a fossil and its surrounding matrix as fossils are often surrounded by or filled with similar-density matrix ( $\mathrm{Wu}$ and Schepartz 2009). To our knowledge, only two reported cases to-date have been successful in recovering fossils from matrix using X-ray CT scans as a guide for preparation (Larkin et al. 2010, Smilg and Berger 2015). In particular, Smilg and Berger (2015), working with Plio-Pleistocene limestone fossils, showed that medical X-ray CT scanning, in addition to being readily available, was also cost-effective and reliable at finding fossils within matrix conglomerates. Here we propose to test the same protocol used by Smilg and Berger (2015) but on a different type of material.

\section{Material and methods}

We X-ray CT scanned two limestone blocks of relatively small size ( $30 \mathrm{~cm}$ wide, 5 and $10 \mathrm{~kg}$ weight), and a third block of larger size ( $70 \mathrm{~cm}$ wide, $80 \mathrm{~cm}$ long, $130 \mathrm{~kg}$ weight). Each block contained several elements of a vertebrate fossil partially exposed at the surface, as well as several exposed invertebrate fossils. The vertebrate fossil, a plesiosaurian specimen, consists of a partial skull and post-cranial elements, the recent discovery of which has already been reported (Mellier and Pouit 2017). The set of analyses presented here are preliminary to the formal, thorough study of this particular fossil. The blocks consisted of a local variant of limestone called 'tuffeau', which is of Cretaceous formation (Turonian age, about 92 million years ago), characterized by a very porous structure, which in itself should facilitate the passage of X-rays through the interior of each block. The blocks were found in a disused, artificially excavated underground quarry in Maine-et-Loire (France).

The X-ray CT scanner used was a Philips Ingenuity CT Family. CT scanning parameters used in helical scan mode were (Tab. 1): tube voltage $140 \mathrm{kV}$, the maximum allowed in medical scans; a tube current of $400 \mathrm{~mA}$ for the two smaller blocks and 1,000 mA for the larger block; matrix size $512 \times 512$ pixels; field of view adjusted according to size of block; axial slice thickness $1 \mathrm{~mm}$. A medical X-ray CT scanner was used due to its availability and cost-effectiveness, and also because it had previously been shown to be successful in the identifying of fossils inside rocks (Smilg and Berger 2015). Image stacks were later prepared in free software InVesalius (Renato Archer Information Technology Center, Campinas, SP, Brazil) and MeshLab (Visual Computing LabISTI CNR).

\section{Results}

X-rays were able to traverse each of the blocks, including the largest one, and image definition, although far from ideal, was sufficient to distinguish many of the objects located within the blocks. Parts of the vertebrate fossil could immediately be distinguished from the surrounding matrix, particularly in the smaller blocks (Text-figs 1,2), with many details of the internal bone structure (Text-fig. 1a, b), teeth (Text-fig. 1b, c) and other skeletal parts (Text-fig. 2a) being apparent. Invertebrate imprints (Text-fig. 1a, c), moulds (Text-fig. 3a, c) and remains of hard skeletons (Textfig. 3a, b), and even the familiar shape of an ammonite (Textfig. $2 \mathrm{a}, \mathrm{c}$ ) were also visible. Radiodensity contrast between

Table 1. CT parameters.

\begin{tabular}{|l|c|c|}
\hline & Block 1 & Block 2 \\
\hline Approximate size $(\mathbf{c m} \times \mathbf{c m} \times \mathbf{c m})$ & $30 \times 30 \times 30$ & $30 \times 40 \times 40$ \\
\hline Weight $(\mathbf{k g})$ & 5 & 10 \\
\hline Scan mode & helical & helical \\
\hline Tube voltage $(\mathbf{k V )}$ & 140 & 140 \\
\hline Tube current $(\mathbf{m A})$ & 400 & 400 \\
\hline Matrix size $($ pixels $\times$ pixels) & $512 \times 512$ & $512 \times 512$ \\
\hline Slice thickness $(\mathbf{m m})$ & 1 & 1,000 \\
\hline
\end{tabular}



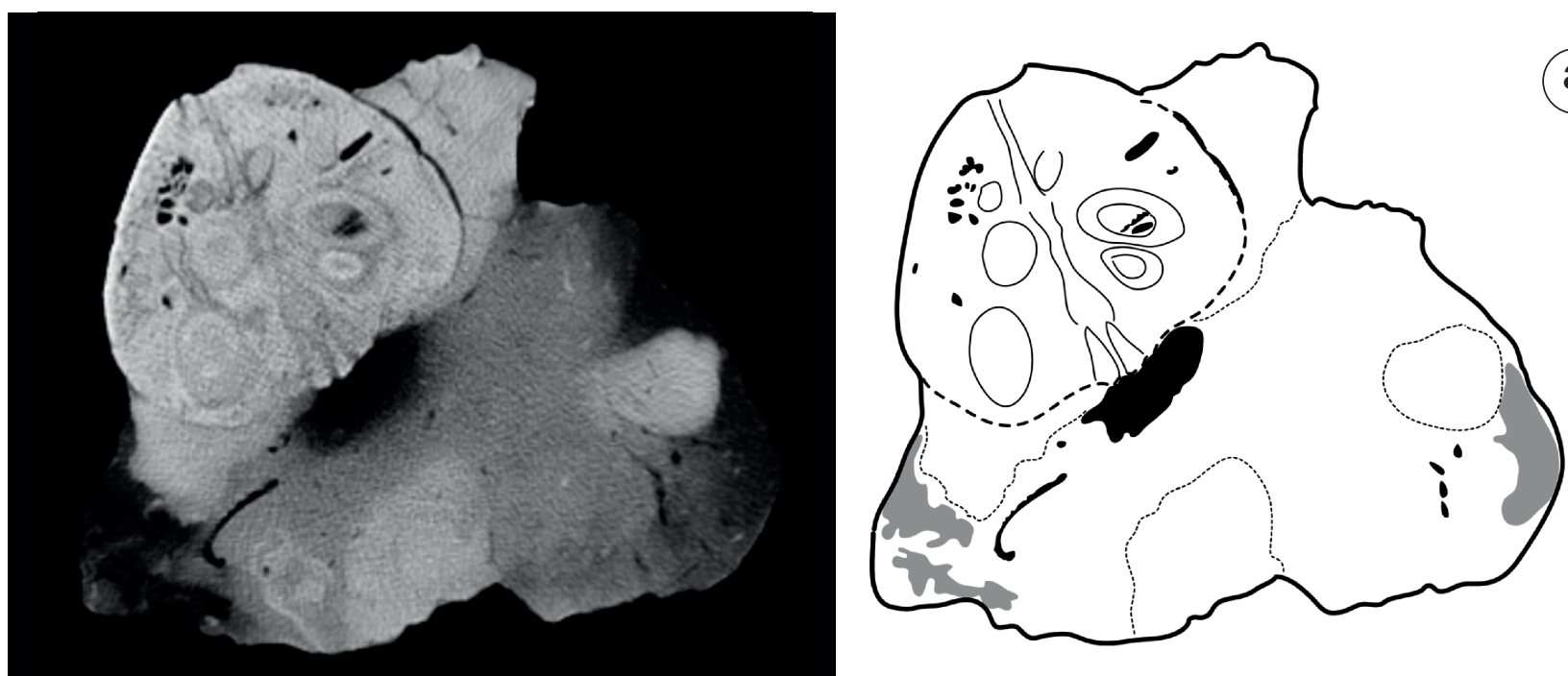

(a)
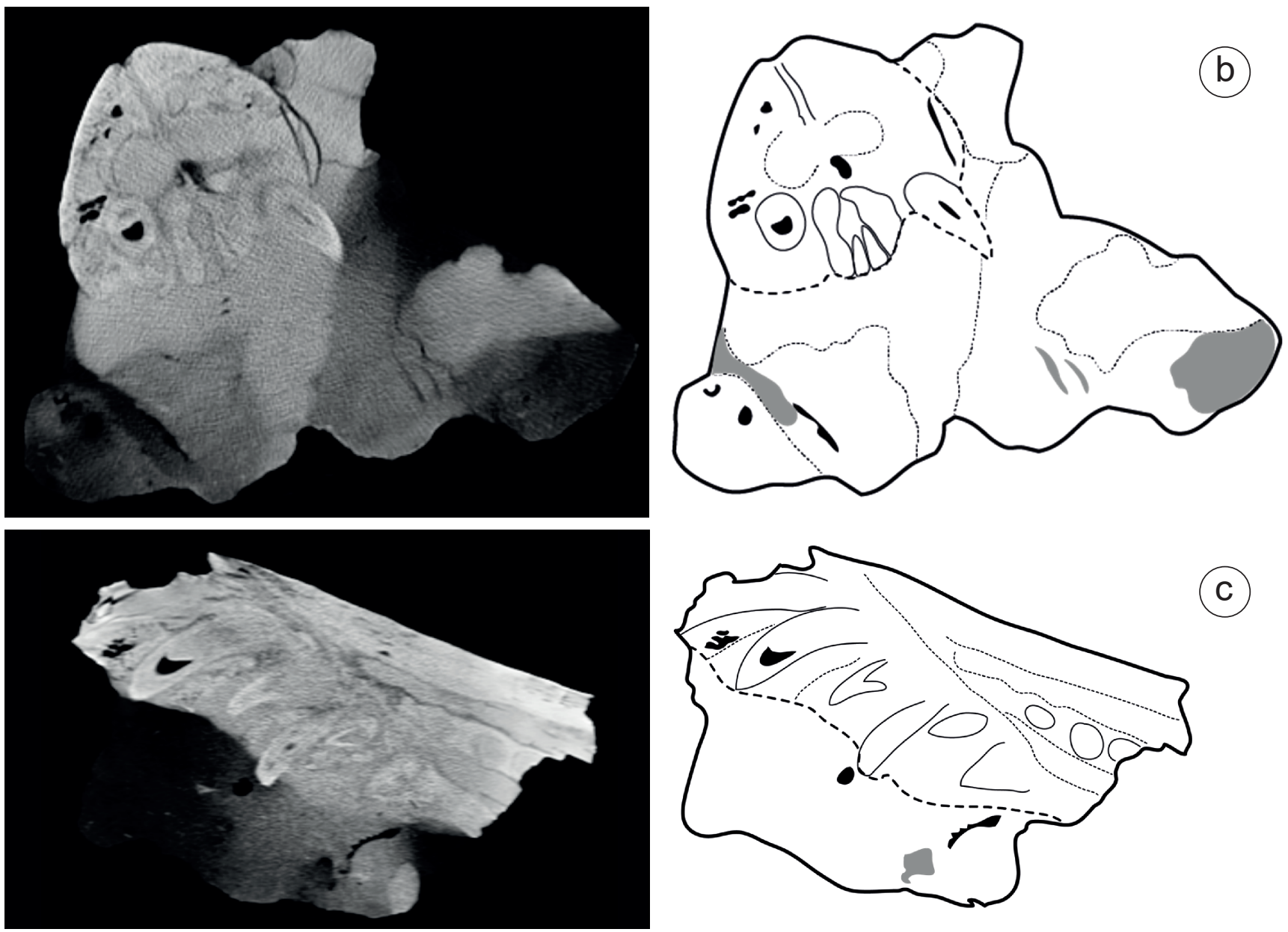

(C)

Text-fig. 1. CT slices on Block 1. Details of the internal bone structure (a, b), teeth (b, c). Invertebrate imprints (a, c). Holes, cracks and empty cavities in both the limestone matrix and within the vertebrate fossil (b).

objects was not always sufficient for visually distinguishing them, but was enough to facilitate the empirical observation of main fossil locations within the block.

The heterogeneity of the 'tuffeau' limestone was also observed, with the more porous (and softer) areas of the matrix being clearly distinguishable from the more compact (and harder) areas by a difference in texture (Text-fig. 2c). This difference between soft and hard areas of the matrix are invaluable information during fossil preparation. Holes, cracks and empty cavities in both the limestone matrix and within the vertebrate fossil were also visible (Text-figs $1 b$, $2 \mathrm{~b}, \mathrm{c})$. Differences in mineral density were easily detected within the calcareous matrix, with highly dense ferric nodules standing out as expected (Text-fig. 2c).

These visual explorations of the CT data were equally important for identifying what could not be seen from the outside. In the largest block (which was cut in the field) large areas of limestone matrix held either only a few scattered invertebrates or no fossils at all (Text-fig. 3b, c). This insight is of the greatest value for speeding up preparation, since 

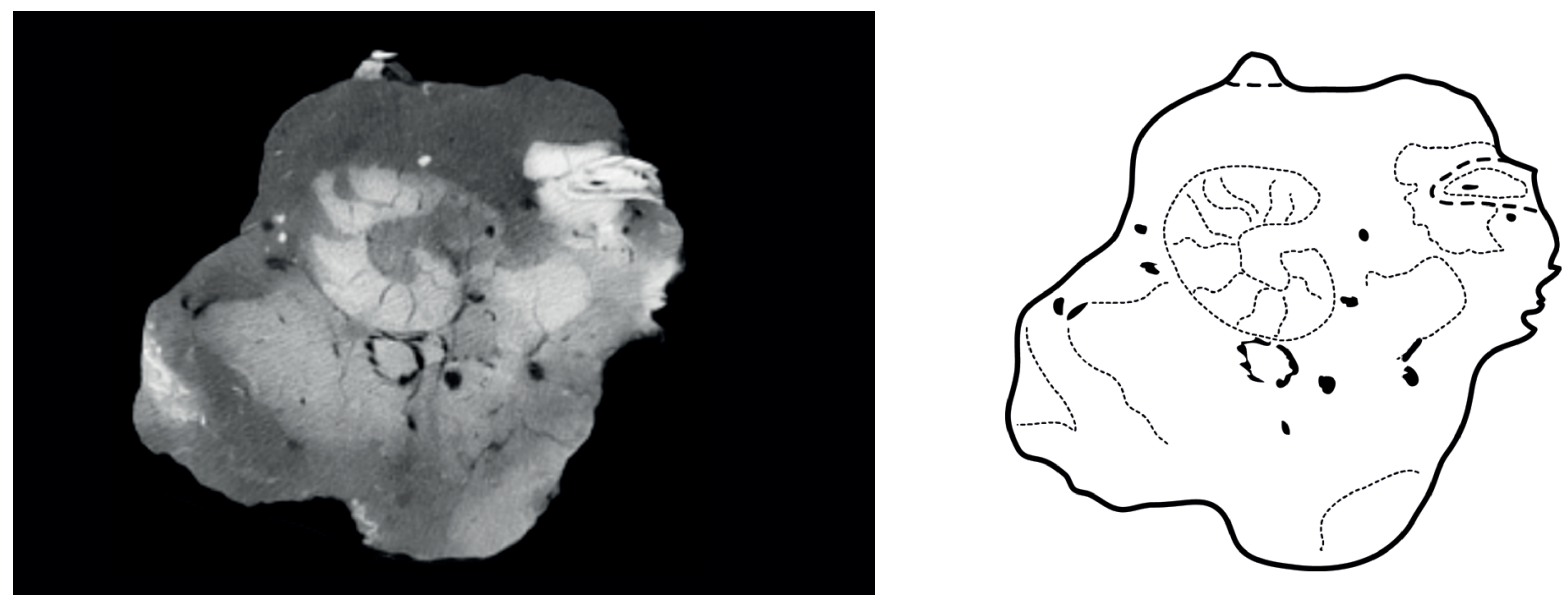

(a)
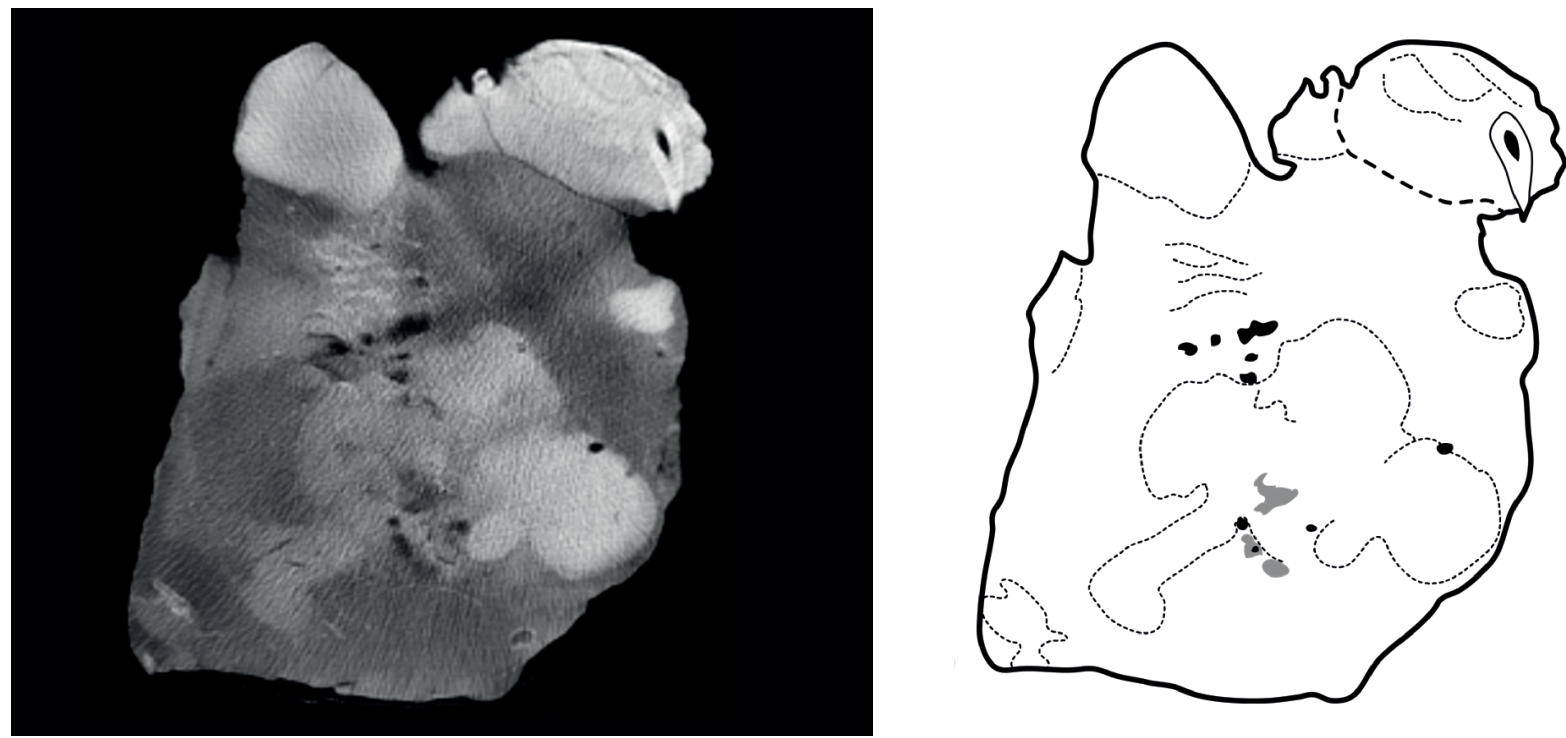

(b)
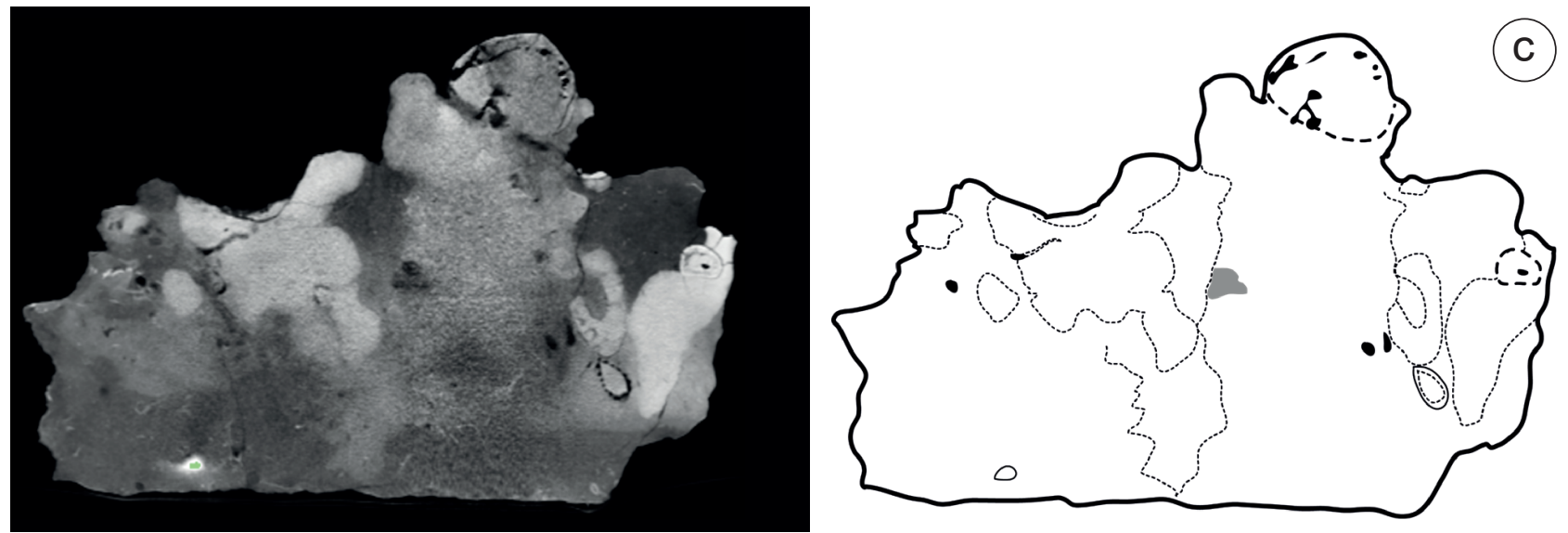

Text-fig. 2. CT slices on Block 2. Details of other skeletal parts (a). The familiar shape of an ammonite (a, c). Holes, cracks and empty cavities in both the limestone matrix and within the vertebrate fossil (b, c). Heterogeneity of the 'tuffeau' limestone, the more porous areas of the matrix clearly distinguishable from the more compact ones (c). Ferric nodules (c).

large parts of the block can be cut out immediately without the need for careful, inch-by-inch exploration. The timeconsuming work can thus be limited to the most important fossil-bearing areas.

\section{Discussion}

In this paper, we explore the use of a medical X-ray CT scanner to inspect blocks of limestone containing vertebrate and invertebrate fossils as an aid to subsequent preparation. Results were largely successful, with low-resolution images and less-than-sharp grey level contrast creating nevertheless sufficient contrast for identifying objects and their location within blocks of limestone. This can subsequently aid physical preparation of the material, and unquestionably optimizes the allocation of time and resources required for preparation.

The substitution of bone calcium by other minerals during diagenesis improved visualization of vertebrate 

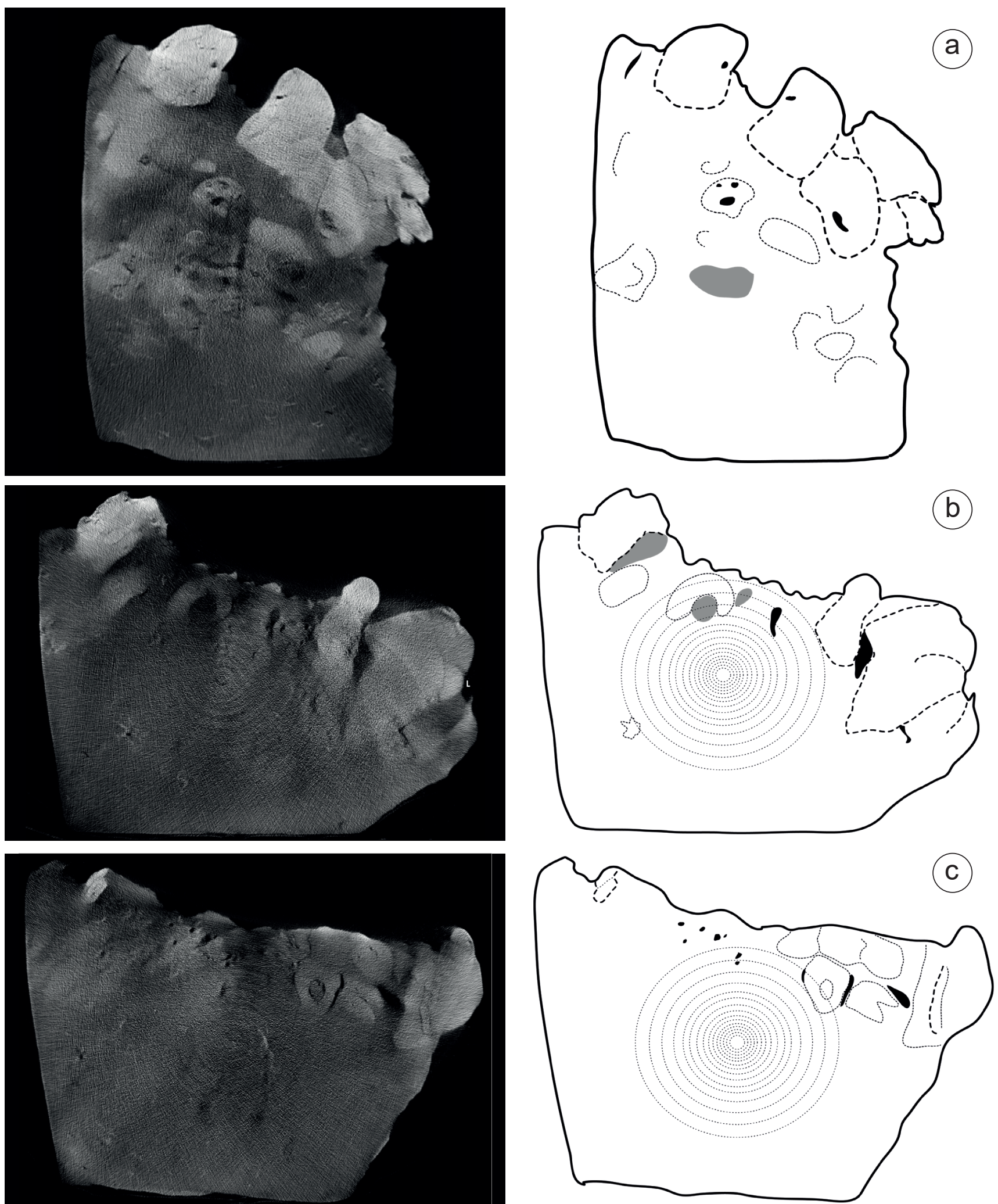

Text-fig. 3. CT slices on Block 3. Invertebrate moulds (a, c) and remains of their hard skeletons (a, b). Large areas of limestone matrix hold either only a few scattered invertebrates or no fossil at all (b, c). Ring artefacts seen close to the isocentre of the scan $(b, c)$ are a well-known phenomenon caused by the X-ray beams traversing the block at an insufficient radiation dose (as expected in such a large block of dense material), and are not part of any physical structure present therein (Triche et al. 2019).

fossils as opposed to the surrounding calcareous matrix due to the metallic compounds contained in these minerals, which have higher density than calcium carbonate. Empty cavities in the limestone possibly indicate places where organic matter has degraded leaving behind an imprint.
Holes may also point to the complex anatomy of vertebrate structures, namely vascular canals, sutures between bones, or pulp cavities in teeth (Text-fig. 1), which were not filled in during fossilization. All unfilled cavities are clearly visible in the X-ray CT scans due to their low radiodensity, 
including invertebrate imprints. The invertebrate remains located close to vertebrate remains are sometimes sacrificed during the manual preparation of the vertebrate specimen in order to save time. The CT data, albeit of a low resolution, can be used to record the existence and location of any invertebrate remains, and may also present an opportunity to discuss whether it would be worthwhile to preserve them or not prior to starting mechanical preparation.

Initially, we had doubts about the capacity of a medical CT scanner to produce sufficiently powerful X-rays which could traverse large blocks of dense matrix, a predicted limitation of such scanners, which are designed to get the best possible image resolution at the lowest dose indicated for causing the least harm to patients. However, the medical machine proved suitable, with X-rays amply able to traverse even the largest block of limestone at the medical CT-level of power. The low resolution of images produced is also a limitation of medical X-ray CT scans, but in our view, it is a minor limitation, since the main purpose here was not so much to produce an accurate digital model of the blocks, but rather a preliminary visual inspection of embedded objects.

Surprisingly, scanning an unprepared block to assist in the subsequent physical preparation of the sample does not appear to be a commonly used technique despite its advantages. Otherwise, if the technique is widely used, that information is seldom reported in publications describing the prepared fossils. This consideration aside, the apparent low use of this protocol could, in our view, be related to three things: 1) many publications deal with specimens discovered decades ago (specimens never studied or re-examined) that were prepared at a time when X-ray CT scanners were not widely available; 2) obtaining data of sufficient quality to be useful to the team of preparators is not systematic, and scanning a big piece of rock is very difficult, possibly involving a complex protocol; 3) the confidence of palaeontologists in their team of preparators (with whom they have often worked for many years) may make this practice unnecessary, costly and time-consuming in their eyes (the physical preparation having been already decided, scanning the fossil would only delay the start of preparation).

Although a digital restoration of the fossils in question could be possible with medical X-ray CT scans, care was taken in not making definitive morphological interpretations from the low-resolution images. If models constructed from scans with the best possible resolution should be treated as satisfactory working hypotheses (Lautenschlager 2016), extra care should be taken when inferring the anatomy from models created out of medical X-ray CT scans. This said, the latter can be satisfactorily used for outreach activities, to supplement museum exhibits, or as a basis for further research (Rahman et al. 2012, Lautenschlager 2016).

In conclusion, use of medical X-ray CT scanners for an initial visual inspection of the interior of limestone blocks for fossil detection is possible, cost effective and reliable. We suggest that physical preparation of limestone blockembedded fossils should only begin after CT scanning is performed, and a standard medical X-ray CT scanner will suffice for this purpose. That way the original raw data is preserved as a digital object (albeit at a low resolution), and physical preparation will not proceed blindly. If X-ray CT is already recognized as an invaluable tool for gaining non- destructive insights into an embedded fossil (Schilling et al. 2014), the advantages of making use of standard medical X-ray CT scanners to facilitate paleontological preparation under logistic or budgetary limitations is becoming more and more apparent.

\section{Acknowledgements}

We would like to thank Professor C. Aubé and his team at the Radiology Department of Angers University Hospital for their enthusiastic openness and commitment toward this project.

The authors warmly thank the anonymous reviewers for their constructive comments which greatly improved an earlier version of this manuscript. We also thank Zuzana Heřmanová (Executive Editor) for editorial support during development of the manuscript.

\section{References}

Balzeau, A., Crevecoeur, I., Rougier, H., Froment, A., Gilissen, E., Grimaud-Hervé, D., Mennecier, P., Semal, P. (2010): Applications of imaging methodologies to paleoanthropology: Beneficial results relating to the preservation, management and development of collections. - Comptes Rendus Palevol, 9: 265-275. https://doi.org/10.1016/j.crpv.2010.07.006

Bristowe, A., Parrott, A., Hack, J., Pencharz, M., Raath, M. (2004): A non-destructive investigation of the skull of the small theropod dinosaur, Coelophysis rhodesiensis, using CT scans and rapid prototyping. - Palaeontologia Africana, 40: 31-36.

Brühl, J. W. (1896): Ueber Verwendung von Röntgen'schen $\mathrm{X}$-Strahlen zu palaeontologischdiagnostischen Zwekken. - Archiv für Physiologie, 1896: 547-550.

Conroy, G. C., Vannier, M. W. (1984): Noninvasive threedimensional computer imaging of matrix filled fossil skulls by high-resolution computed tomography. - Science, 226: 456-458.

https://doi.org/10.1126/science.226.4673.456

Cunningham, J. A., Rahman, I. A., Lautenschlager, S., Rayfield, E. J., Donoghue, P. C. J. (2014): A virtual world of paleontology. - Trends in Ecology and Evolution, 29: 347-357. https://doi.org/10.1016/j.tree.2014.04.004

Donoghue, P. C., Bengtson, S., Dong, X.-p., Gostling, N. J., Huldtgren, T., Cunningham, J. A., Yin, C., Yue, Z., Peng, F., Stampanoni, M. (2006): Synchrotron X-ray tomographic microscopy of fossil embryos. - Nature, 442: 680-683. https://doi.org/10.1038/nature04890

Garcia Sanz, M., Goussard, F., Balzeau, A., Clément, G. (2013): Imaging methodologies in natural sciences: The AST-RX (Acces scientifique a la tomographie a rayons X) Platform of the Muséum d'Histoire naturelle. Paris. - In: Constant, E., Martin, P., Bachau, H. (eds), UVX 2012 11e Colloque sur les Sources Cohérentes et Incohérentes UV, VUV et X; Applications et Développements Récents. EDP Sciences, Les Ulis Cedex, art. no. 01001 (7 pp.). https://doi.org/10.1051/uvx/201301001 
Gee, C. T. (2013): Applying micro CT and 3D Visualization to Jurassic Silicified Conifer Seed Cones: a Virtual Advantage Over Thin-Sectioning. - Applications in Plant Sciences, 1(11): 1300039 (16 pp.).

https://doi.org/10.3732/apps.1300039

Herbin, M., Dupret, V., Goussart, F., Clément, G. (2010): Les techniques d'imagerie 3D au service de la valorisation scientifique des collections anatomiques. - La Lettre a l'OCIM, 131: 13-18.

https://doi.org/10.4000/ocim.105

Hohenstein, P. (2004): X-ray imaging for palaeontology. British Journal of Radiology, 77: 420-425. https://doi.org/10.1259/bjr/27832269

Hounsfield, G. N. (1973): Computerized transverse axial scanning (tomography): I. Description of system. - British Journal of Radiology, 46: 1016-1022. https://doi.org/10.1259/0007-1285-46-552-1016

Jungers, W. L., Minns, R. J. (1979): Computed tomography and biomechanical analysis of fossil long bones. - American Journal of Physical Anthropology, 50: 285-290. https://doi.org/10.1002/ajpa.1330500219

Larkin, N., O'Connor, S., Parsons, D. (2010): The Virtual and Physical Preparation of the Collared Plesiosaur from Bridgewater Bay, Somerset, UK. - The Geological Curator, 9: 107-116.

Lautenschlager, S. (2016): Reconstructing the past: methods and techniques for the digital restoration of fossils. Royal Society Open Science, 3: 160342 (18 pp.). https://doi.org/10.1098/rsos.160342

Lemoine, V. (1896): De l'application des rayons de Röntgen a la paléontologie. - Comptes Rendus Hebdomadaires des Séances de l'Académie des Sciences, Paris, 123: 764-765.

Maldanis, L., Carvalho, M., Ramos Almeida, M., Freitas, F. I., de Andrade, J. A. F. G., Nunes, R. S., Rochitte, C. E., Poppi, R. J., Freitas, R. O., Rodrigues, F., Siljeström, S., Alves Lima, F., Galante, D., Carvalho, I. S., Perez, C. A., de Carvalho, M. R., Bettini, J., Fernandez, V., XavierNeto, J. (2016): Heart fossilization is possible and informs the evolution of cardiac outflow tract in vertebrates. - eLife, 5: 1-12.

https://doi.org/10.7554/eLife.14698

Mellier, B., Pouit, D. (2017): Découverte d'un plésiosaurien dans le Turonien de l'Anjou (Maine-et-Loire). - Bulletin de la Société des Sciences Naturelles de l'Ouest de la France, 39: 59-61.

Pallua, J. D., Kuhn, V., Pallua, A. F., Pfaller, K., Pallua, A. K., Recheis, W., Pöder, R. (2015): Application of microcomputed tomography to microstructure studies of the medicinal fungus Hericium coralloides. - Mycologia, 107(1): 227-238.

https://doi.org/10.3852/14-188

Plessis, A. du, Steyn, J., Roberts, D. E., Botha, L. R., Berger, L. R. (2013): A proof of concept demonstration of the automated laser removal of rock from a fossil using 3D X-ray tomography data. - Journal of Archaeological Science, 40: 4607-4611. https://doi.org/10.1016/j.jas.2013.07.024

Racicot, R. (2017): Fossil Secrets Revealed: X-Ray CT Scanning and Applications in Paleontology. - The Paleontological Society Papers, 22: 21-38.

https://doi.org/10.1017/scs.2017.6
Rahman, I. A., Adcock, K., Garwood, R. J. (2012): Virtual Fossils: a New Resource for Science Communication in Paleontology. - Evolution: Education and Outreach, 5: 635-641.

https://doi.org/10.1007/s12052-012-0458-2

Röntgen, W. C. (1895): Ueber eine neue Art von Strahlen (vorläufige Mittheilung). - Stahel'sche K. Hof- und Universitätsbuch- und Kunsthandlung, Würzburg, 10 pp. (preprint from Sitzungs-Berichte der Physikalisch-medizinischen Gesellschaft zu Würzburg, vol. 137; translated by Arthur Stanton in 1896 under the title On a new kind of rays in Nature, 53(1369): 274-276).

Rowe, T. B., Luo, Z.-X., Ketcham, R. A., Maisano, J. A., Colbert, M. W. (2016): X-ray computed tomography datasets for forensic analysis of vertebrate fossils. Scientific Data, 3: 160040 (25 pp.).

https://doi.org/10.1038/sdata.2016.40

Schilling, R., Jastram, B., Wings, O., Schwarz-Wings, D., Issever, A. S. (2014): Reviving the Dinosaur: Virtual Reconstruction and Three-dimensional Printing of a Dinosaur Vertebra. - Radiology, 270: 864-871. https://doi.org/10.1148/radiol.13130666

Smilg, J. S. (2017): Finding fossils in Malapa breccia medical CT scanning or micro-CT scanning? - South African Journal of Science, 113: 1-6. https://doi.org/10.17159/sajs.2017/20170057

Smilg, J. S., Berger, L. R. (2015): Discovering Hominins Application of Medical Computed Tomography (CT) to Fossil-Bearing Rocks from the Site of Malapa, South Africa. - PLoS ONE, 10(12): e0145340 (19 pp.). https://doi.org/10.1371/journal.pone.0145340

Sutton, M. D. (2008): Tomographic techniques for the study of exceptionally preserved fossils. - Proceedings of the Royal Society, B: Biological Sciences, 275: 1587-1593. https://doi.org/10.1098/rspb.2008.0263

Sutton, M. D., Rahman, I. A., Garwood, R. J. (2014): Techniques for Virtual Palaeontology. Techniques for Virtual Palaeontology. - John Wiley and Sons, London, 208 pp. https://doi.org/10.1002/9781118591192

Sutton, M. D., Rahman, I. A., Garwood, R. J. (2016): Virtual Paleontology - An Overview. - The Paleontological Society Papers, 22: 1-20. https://doi.org/10.1017/scs.2017.5

Tafforeau, P., Boistel, R., Boller, E., Bravin, A., Brunet, M., Chaimanee, Y., Cloetens, P., Feist, M., Hoszowska, J., Jarger, J.-J., Kay, R. F., Lazzari, V., Marivaux, L., Nel, A., Nemoz, C., Thibault, X., Vignaud, P., Zabler, S. (2006): Applications of X-ray synchrotron microtomography for non-destructive 3D studies of paleontological specimens. - Applied Physics, A, 83: 195-202. https://doi.org/10.1007/s00339-006-3507-2

Triche, B. L., Nelson, J. T., Jr., McGill, N. S., Porter, K. K., Sanyal, R., Tessler, F. N., McConathy, J. E., Gauntt, D. M., Yester, M. V., Singh, S. P. (2019): Recognizing and Minimizing Artifacts at CT, MRI, US, and Molecular Imaging. - RadioGraphics, 39: 1017-1018. https://doi.org/10.1148/rg.2019180022

Wu, X., Schepartz, L. A. (2009): Application of computed tomography in paleoanthropological research. - Progress in Natural Science, 19: 913-921.

https://doi.org/10.1016/j.pnsc.2008.10.009 